\title{
PAPR reduction using SC-FDMA in Underwater acoustic channel
}

\author{
Bevek Subba \\ Department of ECE, SRM University, Chennai 603-203, India. \\ G. Elavel Visuvanathan \\ Department of ECE, SRM University, Chennai 603-203, India.
}

\begin{abstract}
Underwater acoustic sensor networks (UWASN) have been developing and attracting growing interests recently. However, underwater acoustic (UWA) communications undergo multipath, delay and limited bandwidth. Orthogonal frequency division multiplexing (OFDM) is suitable for UWA communications because of their immunity against frequency selective fading. It can mitigate inter-symbol interference (ISI). But, OFDM is highly sensitive to the frequency offset, and it also has large peak-to-average power ratio (PAPR). This new modified technique of OFDM is called Single carrier frequency division multiple access (SC-FDMA). SC-FDMA has only single carrier, so it has reduced PAPR. Pulse shaping technique using raised cosine filter reduces PAPR. Based on pulse shaping, the Nyquist filter is used to reduce the PAPR. The complexity and design of this technique is simpler. The bandwidth efficiency is not affected. Also, there is less of In Band as well as Out band distortion. SC-FDMA is applied to UWA channels and MATLAB is used for simulation. For both OFDM and SC-FDMA, Bit error rate (BER) and PAPR will be calculated. The result should show that the less bit error rate than the BER of $10^{-3}$ which is the average can be achieved using SC-FDMA in UWA.
\end{abstract}

Keywords: SCFDMA, Underwater Acoustic Channel, PAPR, Pulse shaping

\section{INTRODUCTION}

UWASN has found an attractive use in a various range of applications, such as military purposes, disaster prevention, remote control in offshore field, pollution monitoring and resource research. There is a need of underwater communications between surface station and underwater sink. Communication between underwater devices is also realized. [1]. The UWA communication channel is complex in nature. The complexity arises due to the inhomogeneous nature of the channel. The various faults are due to salinity, density, temperature changes and inhomogeneity of the water. These are in turn due to the solid or gaseous suspended particles. This are mainly caused by the regular water motion which isn't a problem in aerial. Even the channel perimeter like the bottom and the surface reflects the signal causing multi-path phenomenon which in turn causes ISI and reverberation. The intensity of acoustic wave degrades mainly due to spreading and absorption phenomenon, as it propagates. This is called as transmission loss. Inshore areas got more noise than deep water environment. Ambient Noise affects the Signal to Noise ratio (SNR). Doppler spread is mainly due to the motion of both transmitter and receiver. The range of frequency is decreased. This four aspects are repeatedly varying in nature. The communication system modifies their effects by constantly tracking variations. These factors vary temporarily in the acoustic channel. Thus, making the bandwidth dependent on both distance and frequency. Small bandwidth is present in long range while a short range system have more bandwidth [2].

Underwater environments have low attenuation of sound. OFDM communication has become an attractive modulation technique for UWA communications [3]. The main benefits of OFDM are that it can handle the multi-path interference, and mitigate inter-symbol interference (ISI) caused by the multipath. It divides the available bandwidth into subcarriers and in the process causes bit error rates in frequency selective fading. Channel coding plays a vital role on the performances of OFDM based wireless system. It provides high data rates communication over multipath channels [2]. But, OFDM is erroneously sensitive to the Doppler variation, sampling deviation and have high PAPR.

DFT-precoded version of OFDM is called as SC-FDMA. It has instigated lot of interests in wireless communication because of its reduced PAPR effects. In transmitter side, it uses single carrier modulation and in the receiver side, frequency domain equalization respectively. SC-FDMA signal has single carrier structure. Power efficiency and less implementation cost are the advantages of low PAPR. Another advantage is that data detection is performed in time domain.

\section{PULSE SHAPING BASED SC-FDMA TRANSCEIVER}

In SC-FDMA (DFT spreading FDMA), DFT converts the symbols from time domain into frequency domain as understood. Than the modulator converts the input sequence into a complex numbers sequence. This $\mathbf{M}$ modulated symbols are grouped into each block. Then, each of the output of DFT will be corresponding to one of the $\mathrm{N}$ subcarriers. In SCFDMA, number of subcarriers $\mathrm{N}$ must be greater than symbols M. 
The outputs of DFT is allocated to the whole bandwidth and the unallocated subcarriers is occupied by zeros, in the distributed subcarrier mode. The interleaved SC-FDMA (IFDMA) is more preferable than the localized mode because IFDMA is superior in terms power efficiency [8]. Whereas in the IFDMA, to avoid adjacent interference, subcarriers are divided equally over the entire bandwidth. Towards the end, subcarrier is converted back to complex time domain signal by inverse FFT (IFFT) [4].

The ISI has to be removed from IFFT signal. For that case, cyclic prefix (CP) is added in which cyclic convolution is used. A pulse shaping which is also called as linear filtering, is used for PAPR reduction to suppress the out band signal [10]. Then, the modulated subcarriers is convolved with the filters impulse response. Pulse shaping (PS) is needed in order to bandlimit. But, the PAPR is increased in the process [4].

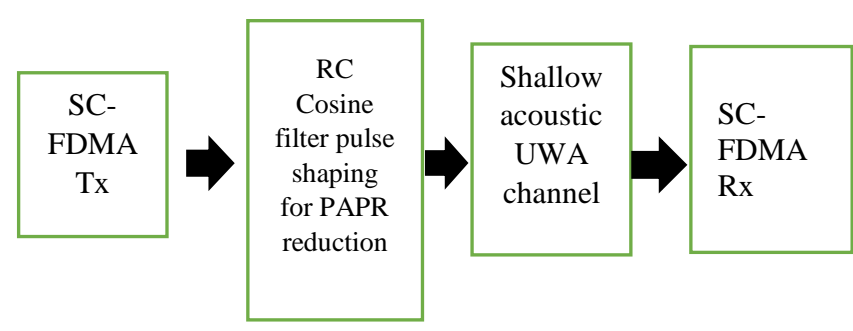

Figure 1: Proposed block model

\section{MULTIPATH UWA CHANNEL}

The bandwidth for acoustic is limited which is better supported at low frequencies. Frequency of an acoustic system ranges from $10 \mathrm{kHz}$ to $15 \mathrm{kHz}$. The bandwidth is $5 \mathrm{kHz}$ which is low, but actually it is in fact ultra-wideband. $1500 \mathrm{~m} / \mathrm{s}$ is the speed of sound underwater as compared to the aerial. Delay spreading in a long term causes a frequency selective distortion and motion under water causes a Doppler effect. This can lead to reverberation and ISI. UWA channel has the combination of low physical link quality and high latency. Reflection and refractions are the main cause of multipath formation. The multipath formation can be through surface and bottom of the channel [5].

UWA channel has the surface and bottom reflection properties. The geometry of the channel and its reflection properties are the two factors on which the impulse response depends. These determines the number of multiple propagation paths, delays and strengths. Those that have went through multiple reflections are discarded as they lost much of energy. Let us denote the length by $l_{p}$ of the $p$-th propagation path, whereby $p$ $=0$ corresponds to the propagation path arrived first. In shallow depth, the sound speed is constant $c$, plain geometry is used to calculate path lengths and path delays is $l_{p} / c$. The arrival time of strongest path is value $t_{0}$. It calculates the reference time at the destination. Path delays can be given as $\tau_{p}=l_{p} / c-t_{0}$ [5].

Under ideal condition, value of Surface reflection coefficient $\Gamma_{\mathrm{p}}$ is -1 . Bottom reflection coefficients depends on the smoothness of surface and also the angle between the incident and the horizontal axis i.e. grazing angle. $\Gamma_{\mathrm{p}}$ is the cumulative reflection coefficient and $A_{p}$ is the propagation loss associated with that pth path. The gain of this path will be $g_{p}=\Gamma_{\mathrm{p}} / \widehat{ } A_{p}$. The channel response usually for a radio channel can be expressed as,

$$
g(t)=\Sigma g_{p} \delta\left(t-\tau_{p}\right)
$$

The path gain depends on frequency in an acoustic environment. Therefore, only for a single frequency tone, it is constant. Each frequency will undergo an attenuation for a broadband signal. With the attenuation, the $p$-th path frequency response is,

$$
G_{p}(f)=\Gamma_{p} / \sqrt{ } A\left(l_{p}, f\right)
$$

So, the path is a low pass filter, with its own dispersion. In the frequency domain, the overall channel response is

$$
G(f)=\Sigma G_{p}(f) \exp \left(-j 2 \pi f \tau_{p}\right)
$$

and the impulse response is

$$
g(t)=\Sigma g_{p}\left(t-\tau_{p}\right)
$$

where $g_{p}(t)$ is the inverse Fourier transform of $G_{p}(f)$ [5].

\section{MATHEMATICAL MODEL FOR UWA}

The acoustic path loss is [6]:

$$
A(l, f)=A_{o} l^{k} \alpha(f)^{l}
$$

Where $k$ is the spreading factor, 1 is the distance, $A_{0}$ is a unitnormalizing constant and $\alpha(f)$ is the absorption coefficient. Using Thorp's empirical formula, the absorption coefficient can be given as [6]

$a(f)=0.11 \frac{f^{2}}{1+f^{2}}+44 \frac{f^{2}}{4100+f^{2}}+2.75 \times 10^{-4} f^{2}+0.003$

Where $f$ is given in $\mathrm{kHz}$ and the absorption coefficient is given in $\mathrm{dB} / \mathrm{km}$. The attenuation and cumulative reflection coefficient are summed together. Therefore, in frequency domain, the overall transfer function can be denoted as follows [5]:

$$
G(l, f)=\sum_{p=0}^{P-1} \frac{\Gamma_{p}}{\sqrt{\left(A\left(l_{p}, f\right)\right)}} e^{-j 2 \Pi f \tau_{p}}
$$

Where $\tau_{p}=l_{p} / c$ is the path delay and $c$ is the speed of the sound underwater $(1500 \mathrm{~m} / \mathrm{s})$. For paths each of length $l_{p}$, $p=0 \ldots P-1, \Gamma_{p}$ shows additional losses on the $p^{\text {th }}$ path and is given as follows [6]:

$$
\Gamma_{p}=\gamma_{s}^{n_{s p}} \gamma_{p}^{n_{b p}}\left(\theta_{p}\right)
$$

Where $\theta_{\mathrm{p}}$ is the grazing angle between the received signal and the horizontal axis associated with the $\mathrm{p}^{\text {th }}$ path, $\gamma_{\mathrm{s}}$ is the surface reflection coefficient and $\gamma_{p}$ is the bottom reflection coefficient. $\mathrm{n}_{\mathrm{sp}}$ and $\mathrm{n}_{\mathrm{bp}}$ being the number of surface and bottom reflections. Each bottom reflection is modeled by a coefficient $\gamma_{b}(\theta)[6]:$ 
$\gamma_{b}(\theta)=\left\{\begin{array}{cl}\frac{\rho_{b} \sin (\theta)-\rho \sqrt{\left(\frac{c}{c_{b}}\right)^{2}-\cos ^{2}(\theta)}}{\rho_{b} \sin (\theta)+\rho \sqrt{\left(\frac{c}{c_{b}}\right)^{2}-\cos ^{2}(\theta)}}, \quad \cos (\theta) \leq c / c_{b} \\ 1, \text { otherwise }\end{array}\right.$

Where $\rho$ and $c$ are the density and speed of sound in water respectively. $\rho_{b}$ and $c_{b}$ are the density and speed of sound in bottom [6].

The ambient noise is Gaussian distributed because it is random and the distribution are not known. Considering a fixed carrier frequency $f$ and around it a band of frequencies of width $\Delta f$, SNR is calculated by:

$$
S N R=P D / A \cdot N
$$

where PD is the power spectral density, whose power is dependent on the distance, $\mathrm{N}$ is ambient noise and $\mathrm{A}$ is acoustic path loss [5].

\section{NOISE CONSIDERATION IN UWA CHANNEL}

Ambient noise is a kind of a background noise present in UWA channel. Depending on the frequency, ambient noise can be turbulence noise, shipping noise, wind noise and thermal noise [6] described in Table I.

Table I: Ambient Noise

\begin{tabular}{|l|l|l|}
\hline Types & Frequency & Cause \\
\hline Turbulence noise & $0.1 \mathrm{~Hz}-10 \mathrm{~Hz}$ & Water motion \\
\hline Shipping noise & $10 \mathrm{~Hz}-100 \mathrm{~Hz}$ & Ship traffic \\
\hline Wind noise & $1 \mathrm{kHz}-30 \mathrm{kHz}$ & Wind and rain \\
\hline Thermal noise & $+100 \mathrm{kHz}$ & $\begin{array}{l}\text { Water molecules } \\
\text { vibration }\end{array}$ \\
\hline
\end{tabular}

Since my project deals with frequency $10 \mathrm{kHz}$ to $15 \mathrm{~Hz}$ for a shallow water, wind noise can be considered. Wind noise can be calculated by:

$10 \log N(f)=50+7.5 \sqrt{\mathrm{W}}_{\mathrm{W}}+20 \log (f)-40 \log (f+0.4)$

where $w$ is the wind speed in $\mathrm{m} / \mathrm{s}[6]$.

\section{PAPR REDUCTION USING RAISED COSINE FILTER}

The PAPR is the ratio between the maximum power to the average power. PAPR is different at separate instant of phase values. A peak in the output envelope is caused when all the points obtain the maximum value consecutively [9]. The peak value of the system is very high due to numerous modulated subcarriers. It is the fluctuation of peak power or instantaneous power from the mean. Peak can swing and crosses into nonlinear region, orthogonality is lost. This peaks leads to amplification inefficiency and cost becomes high. It causes saturation in power amplifier. In the process, the complexity of both analog to digital and digital to analog converter is well increased [7].

Peak power is $\mathrm{N}$ times greater than the average power. OFDM signals are highly sensitive. That's because of the nonlinearity of the power amplifier. Each symbol is modulated to each of a group of subcarriers, $(k=0,1, \ldots \ldots, N-1)$. In the process, a block of $N$ symbols $(X)$ is generated. The resulting signal is given as:

$$
x(t)=\sum_{n=0}^{N-1} X_{k} e^{j 2 \Pi f_{k} t},
$$

PAPR is defined as,

$$
P A P R=\frac{\max |x(t)|^{2}}{\left[E|x(t)|^{2}\right]}
$$

where $E[$.$] denotes the expectation operator.$

The PAPR can be handled by both modulation and pulse shaping. The Nyquist pulses have different decay rates. Its decay rate has a huge effect on PAPR. PS filter's two biggest sidelobes causes the PAPR to increase. The reduced size tail of the side lobe of the SC-FDMA can reduce the PAPR [7].

Raised Cosine Pulses are the modified sinc pulses. It has a bandwidth of $W$ where $W=1 / 2 T_{s}$. It has an adjustable bandwidth which varies from $W$ to $2 W$. The achieved bandwidth is related to the ideal bandwidth as $\alpha=1-W / W_{o}$ where $\alpha$ is the roll-off factor, $W$ is the Nyquist bandwidth and $W_{o}$ is the utilized bandwidth. The roll off factor value ranges from 0 to 1 . The raised cosine pulse in the time domain is [11]:

$$
h(t)=\frac{\sin \frac{2 \Pi t}{T s}}{2 \Pi t} \frac{\cos 2 \Pi \alpha t}{1-\left(\frac{2 \alpha t}{\Pi}\right)^{2}}
$$

Sinc pulse is the first part. Whereas, the second part is the cosine correction of the sinc pulse. The Nyquist Bandwidth is greater by a factor of $(1+\alpha)$. Bandwidth depends on the roll-off factor $\alpha$. For $\alpha=0$ it becomes a sinc pulse and for $\alpha=1$, it becomes that of pure square pulse [11]. The frequency domain relationship is given by [11]:

$$
H(f)=\left\{\cos ^{2} \frac{\Pi T_{S}}{2 \alpha}\left[|f|-\frac{(1-\alpha)}{2 T_{S}}\right]\right\} \begin{array}{r}
\text { for }|f| \leq \frac{1-\alpha}{2 T_{S}} \\
\text { for } \frac{1-\alpha}{2 T_{S}} \leq|f| \leq \\
\text { for }|f|>\frac{1+\alpha}{2 T_{S}}
\end{array}
$$

\section{SIMULATION RESULTS AND DISCUSSION}

Simulations in MATLAB was done to study the PAPR performance and SNR characteristics considering ambient noise and path loss. Pulse shaping used to obtain better PAPR performance. 
Table II: Simulation Parameters

\begin{tabular}{|l|l|}
\hline Bandwidth & $5 \mathrm{kHz}$ \\
\hline Frequency & $10 \mathrm{kHz}$ \\
\hline Transmission distance & $3 \mathrm{~km}, 5 \mathrm{~km}$ and 10km \\
\hline Size of the block $\mathrm{N}_{\mathrm{d}}$ & 480 \\
\hline No. of subcarriers $\mathrm{N}$ & 2400 \\
\hline Subcarrier spacing & $2.3 \mathrm{~Hz}$ \\
\hline Duration of data block T & $434 \mathrm{~ms}$ \\
\hline CP duration $\mathrm{T}_{\mathrm{cp}}$ & $16.8 \mathrm{~ms}$ \\
\hline Spreading factor $\mathrm{k}$ & 1.5 \\
\hline Roll off factor & $0,0.5,1$ \\
\hline $\begin{array}{l}\text { Surface reflection } \\
\text { coefficient } \Gamma_{\mathrm{s}}\end{array}$ & -1 \\
\hline $\begin{array}{l}\text { Number of surface and } \\
\text { bottom reflection }\end{array}$ & 1 \\
\hline
\end{tabular}

The CCDF is the probability that the calculated PAPR of the output signal exceeds a threshold value. So, it is 1-CDF. As, rolling factor increases, PAPR reduces and reduced tail size. Further the curve towards the right, it has high PAPR.

$10 \%$ probability that PAPR is greater than $6.3 \mathrm{~dB}$ for $\mathrm{SC}$ FDMA (rolling factor 1) compared to rolling factor 0.5 whose $10 \%$ probability that PAPR is greater than $7.1 \mathrm{~dB}$. There is more deviation for rolling factor for rolling factor 0.5 than rolling factor 1 from $0 \mathrm{~dB}$. So, comparatively with rolling factor 1 has better PAPR reduction than OFDM. The maximum reduction in PAPR value of about $8 \mathrm{~dB}$ is achieved by SC-FDMA.

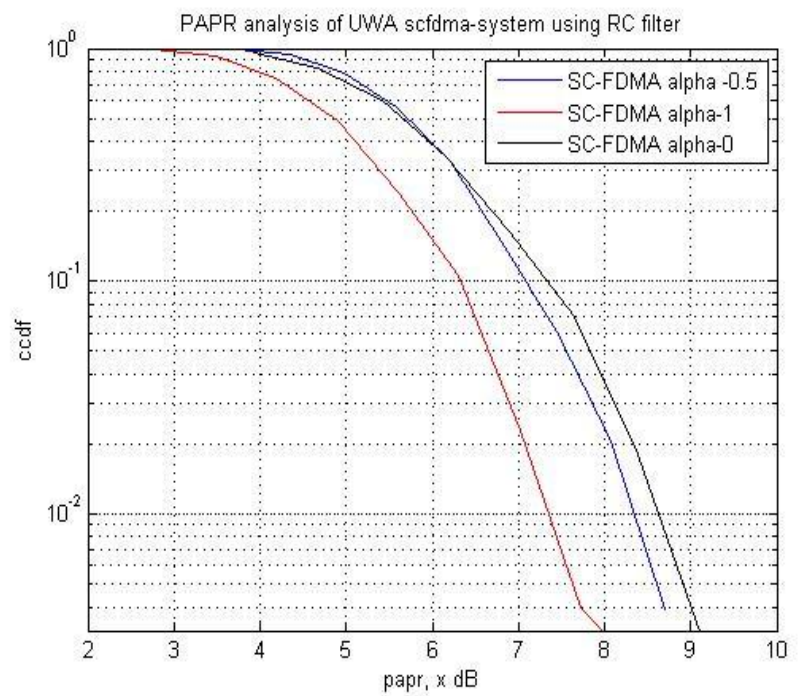

Figure 2: PAPR of UWA SCFDMA system
Table III: 3 Multipath UWA Channel Analysis for Frequency 10khz.

\begin{tabular}{|c|c|c|c|c|}
\hline $\begin{array}{c}\text { Transmis- } \\
\text { sion length } \\
\quad \mathbf{l}(\mathbf{k m})\end{array}$ & $\begin{array}{c}\text { Path } \\
\text { delay } \\
\tau(\mathrm{s})\end{array}$ & $\begin{array}{c}\text { Path } \\
\text { loss A } \\
\text { (dB) }\end{array}$ & $\begin{array}{l}\text { Absorption } \\
\text { coefficient }\end{array}$ & $\begin{array}{c}\text { Path } \\
\text { gain } \\
(\boldsymbol{\Gamma p} / \sqrt{\mathbf{A}})\end{array}$ \\
\hline 3 & 2 & 19.1 & \multirow{3}{*}{$\begin{array}{c}1.203 \\
\mathrm{~dB} / \mathrm{km}\end{array}$} & 0.33 \\
\hline 5 & 3.33 & 28.99 & & 0.19 \\
\hline 10 & 6.66 & 46.1 & & 0.071 \\
\hline
\end{tabular}

Table IV: 3 Multipath UWA Channel Analysis for Frequency $15 \mathrm{khz}$.

\begin{tabular}{|c|c|c|c|c|}
\hline $\begin{array}{c}\text { Transmis- } \\
\text { sion length } \\
\text { l (km) }\end{array}$ & $\begin{array}{l}\text { Path } \\
\text { delay } \\
\tau(s)\end{array}$ & $\begin{array}{c}\text { Path } \\
\text { loss A } \\
(\text { dB })\end{array}$ & $\begin{array}{c}\text { Absorption } \\
\text { coefficient }\end{array}$ & $\begin{array}{c}\text { Path } \\
\text { gain } \\
(\boldsymbol{\Gamma p} / \sqrt{\mathbf{A}})\end{array}$ \\
\hline 3 & 2 & 38.19 & \multirow{3}{*}{$2.5 \mathrm{~dB} / \mathrm{km}$} & 0.16 \\
\hline 5 & 3.33 & 60.76 & & 0.13 \\
\hline 10 & 6.66 & 109.59 & & 0.095 \\
\hline
\end{tabular}

In Table II, path gain and path loss was analyzed for frequency $10 \mathrm{kHz}$ for $3 \mathrm{~km}, 5 \mathrm{~km}$ and $10 \mathrm{~km}$ respectively. The absorption coefficient is $1.203 \mathrm{~dB} / \mathrm{km}$ calculated from equation (6). We know that in a UWA channel as transmission length increases, path gain decreases. Similarly in Table III, it was calculated and studied for frequency $15 \mathrm{kHz}$ with the increased absorption coefficient $2.5 \mathrm{~dB} / \mathrm{km}$.

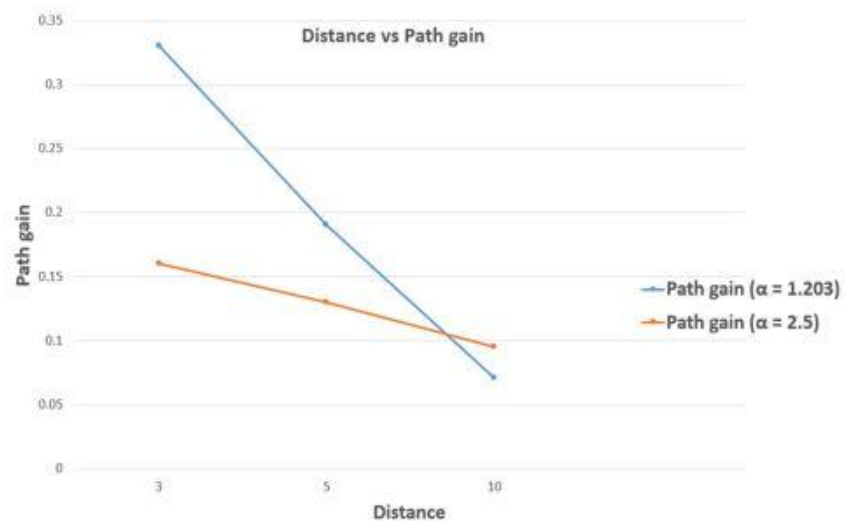

Figure 3: Distance vs Path gain for different absorption coefficient

In figure 3 , I have plotted the graph between distance and path gain for absorption coefficient 1.2 and 2.5. When absorption coefficient increases path gain decreases but for $10 \mathrm{~km}$ distance, the path gain increases. For absorption coefficient 1.203, path 
gain decreases drastically but for 2.5 , its not a drastic decrease rather it's a slow decrease.

From figure 4, we can conclude that BER remains constant for increase of SNR till 5dB. Than after that it starts decreasing.

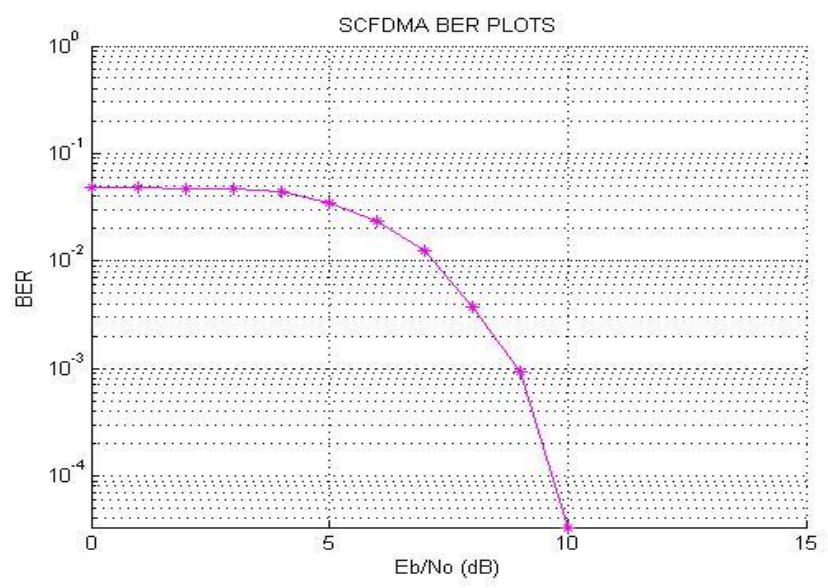

Figure 4: BER vs SNR for SC-FDMA UWA

\section{CONCLUSION}

UWA is a complicated channel due to its bandlimited and reverberant nature. SC-FDMA can be an appropriate solution for UWA channel which takes the advantage of OFDM to handle multipath and also the reduction of PAPR. To reduce the PAPR of the system, pulse shaping is used. The complexity and design of the technique is simpler as only a simple RC filter is used. There is very low in band as well as low out band distortion. This technique doesn't affect the bandwidth efficiency of the system.

\section{REFERENCES}

[1] I. F. Akyildiz, D. Pompili, and T. Melodia, "Underwater acoustic sensor networks: Research challenges", Ad Hoc Networks Journal, (Elsevier), 3(3):257-279, March 2005.

[2] Pallavi Suryawanshi, Vaishali Sonone, Ashish Jadhav, "Underwater Communication by using OFDM system", International Journal of Scientific and Research Publications, Volume 3, Issue 12, December 2013.

[3] Lanbo Liu, Shengli Zhou, and Jun-Hong Cui," Prospects and Problems of Wireless Communication for Underwater Sensor Networkss", WILEY WCMC SPECIAL ISSUE ON UNDERWATER SENSOR NETWORKS (INVITED).

[4] Zhuoming Feng, Xiongfei Tao, Weizhong Liu, and Zuoqi Hu, "PAPR Reduction in SC-IFDMA Using a Piece-Wise Linear Nyquist Filter", IEEE communications letters, vol. 19, no. 3, March 2015.
[5] M. Stojanovic and J. Preisig, "Underwater acoustic communication channels: Propagation models and statistical characterization", IEEE Communications Magazine, 47(1):84-89, January 2009.

[6] Aser M. Matarneh, "Thorough Investigation of BER Simulation of DPSK in Underwater Acoustic Channel", Jordan Journal of Electrical Engineering, Vol. 2 Pages 160-171, no. 2, 2016.

[7] V. Vijayarangan \& R. Sukanesh, "Reduction of PAPR in OFDM using Pulse shaping Techniques, International Journal of Electronics Engineering, 1(1), 2009.

[8] Xilin Cheng, Fengzhong Qu, and Liuqing Yang, "Single Carrier FDMA over Underwater Acoustic Channels", 6th International ICST Conference on Communications and Networking in China (CHINACOM), 2011

[9] Arun Gangwar, Manushree Bhardwaj, "An Overview: Peak to Average Power Ratio in OFDM system \& its Effect", International Journal of Communication and Computer Technologies Volume 01 - No.2, Issue: 02 September 2012.

[10] Suverna Sengar, Partha Pratim Bhattacharya, "Performance Improvement in OFDM System by PAPR Reduction Using Pulse Shaping Technique", International Journal of Emerging Technology and Advanced Engineering, ISSN 2250-2459, ISO 9001:2008 Certified Journal, Volume 2, Issue 12, December 2012.

[11] Proakis, Digital Communications, 4th-ed-2, page $543-$ 550 